\title{
Dialogue Between Sincerity and Piety: A Study on the Artistic Characteristics of Documentary Photography Works of Lu Nan's Four Seasons: Daily Life of Tibetan Peasants
}

\author{
$\mathrm{Xu}$ Wenhao ${ }^{1, \mathrm{a}}$ \\ ${ }^{1}$ College of Fine Arts, Hunan Normal University, Yuelu District, Changsha, Hunan, China \\ 563492566@qq.com
}

\begin{abstract}
This paper focuses on the artistic expression of lu Nan's documentary photography "Four Seasons: The Daily Life of Xizang Farmers", and explores the visual expression and spiritual connotation of the image to obtain the unique value orientation of the "precipitation type" photographer by combining the analysis of the material media of the photos and a large number of investigations on the background of the photographer's creation.In the photography collection, the aesthetic sense of visual form of the photos presents a dual relationship. In the composition, the central composition of Oriental ceremony and the linear composition of western oil painting interweave and coexist, and the plain black tone and the holy white tone accompany each other.This dual relationship, along with the "interest" of photography of Tibetan national spirit in Tibet, triggers the audience to think about the "thorn" of photography of life and death.Based on the visual analysis of this individual photographer, this paper discusses the various possibilities of visual analysis, and arouses more attention to the visual exploration of local photographers.
\end{abstract}

Keywords: Lu Nan, Tibetan farmers, Composition aesthetics, Tone aesthetics, Philosophy of photography

\section{真诚与虔诚的对话: 吕楠《四季: 西藏农民的日常生活》 纪实摄影作品艺术特色探究}

\author{
徐文昊 ${ }^{1, a}$
}

${ }^{1}$ 湖南师范大学美术学院, 岳麓区, 长沙市, 湖南省, 中国 a563492566@qq.com

\section{摘要}

本文着重讨论吕楠的《四季: 西藏农民的日常生活》纪实摄影作品的艺术表现, 结合照片物质媒介分析及对摄 影师创作背景的大量调研, 探究影像的视觉表现以及精神内涵得出 “沉淀式” 摄影师所特有的价值取向。在摄 影集中, 照片视觉形式美感呈现了一种双重关系，构图中的东方仪式中心构图与西方油画感的线性构图交织并 存, 影调的质朴的黑色调与圣洁的白色调如影相随。这种双重关系随着西藏藏民的民族精神的摄影 “意趣” ， 引发观者对生命与死亡这一摄影 “刺点” 思考。基于这一摄影师个例视觉分析, 探讨了视觉分析的多样可能, 引发更多对本土摄影师视觉探究的关注。

关键词: 吕楠；藏族农民；构图美学；影调美学；摄影哲学 


\section{1. 引言}

《庄子》中的 “真者, 精诚之至也, 不精不诚, 不能动人”, 正是对吕楠的摄影最好的注释, 他用真 诚创作最摄人心魄的纪实影像。吕楠的摄影生涯并不 算是多产，在辞去《民族画报》工作后成为自由摄影 师。而他通过 15 年时间沉淀下来的《被遗忘的人: 精神病人生存现状》《在路上: 中国的天主教》和《四 季：西藏农民的日常生活》三部曲是对人生不同生命 状态的写照。从《被遗忘的人》身体的痛苦, 到《在 路上》灵魂的净化, 最后实现《四季》心灵的平静祥 和是吕楠通过镜头对人生的解读。

《四季: 西藏农民的日常生活》作为三部曲中的 “升华” , 为了得到西藏藏民这一中国大地上的神秘 文化形态的视觉表现, 他潜伏到淳朴的农村大地, 探 究虔诚的西藏农民心中纯洁的圣光, 利用手中的相机 实现了真诚摄影与虔诚生活的对话, 最终得到了散发 着人性光辉的视觉图像。

\section{2. 《四季》作品艺术特色探究}

吕楠的《四季》主要记录的是 21 世纪初中国西 藏农村大地上藏族农民的日常活动, 该作品聚焦于中 国西藏农民这一极其神秘且纯洁的群体, 却并未直面 西藏佛事宗教宏伟教化的精神属性, 而是从日常生活 细节挖掘中国少数民族中农民的人性光辉。栗宪庭十 分欣赏吕楠的作品, 认为他拍摄的照片象征了人类现 如今的精神状况, 象征了人类伟大精神的复归。 ${ }^{[1]}$ 在 吕楠的整部摄影作品中看不到 “模式化” 精准搭配的 人物关系与构图, 也没有人云亦云的观念介入, 他通 过快门将自己的风格凝固于照片之中, 最终沉淀为属 于自己的精神符号。

\section{1 吕楠摄影构图美学}

构图作为摄影师对拍摄题材及画面的选择, 能充 分体现摄影师对拍摄对象的感悟与艺术表达。吕楠在 整本影集中并不是限制于某一固定构图方式, 也不刻 意强调黄金比例, 而是巧妙将自己对生活和民族精神 哲学的感悟融入自己的构图画面中, 从而使画面达到 超凡脱俗的意境。在画面构图上给观者最大的审美体 验是东方传统生活仪式感与西方油画线性描绘的和 谐共生。

\subsection{1 构图中的仪式感}

摄影自西方传入中国以来, 长久的担任着肖像记 录的职责, 也潜意识的将这种生活仪式刻入到中国传 统的思维中。这种仪式感是我们面对相机时, 会习惯 性的端庄坐姿, 使得自己保持良好的体态, 在最终的 照片中展示一种无可挑剔的尊严。正如苏珊 - 桑塔格 在《论摄影》中提到: 在中国, 拍照永远是一种仪式, 永远涉及摆姿势, 当然还需要征得同意。如果某个人
“故意捕捉一些不知道他来意的群众镜头”，则他无 异于剥夺人民和事物摆出最好看的姿势的权利。 ${ }^{[2]}$ 在 吕楠这部作品中，同样大量运用中心式构图对中国西 藏农民进行肖像记录。《外祖母和外孙女》是拍摄于 2001 年, 照片的主角是藏族外祖母和她的外孙女。 画面中的血缘关系的亲密属性通过外祖母双手环抱 女孩所体现，照片中的被摄者俨然将摄影视为生活的 一种仪式, 面对镜头的奇袭, 刻意维持着自己身体的 端庄姿态，又不可避免的在形体上显得僵硬。将民族 性的元素从画面抽离, 这样一幅照片与中国传统家庭 记录的仪式照片并无差异。吕楠作为土生土长的中国 摄影师拍摄中国西藏农村大地的藏民, 在定格的照片 中无时无刻不流露着中国传统价值观念的影响, 也正 是在吕楠这样构图美学的纪实摄影中, 让我们认识到 中华大地上的另一种生活形式。

\subsection{2 构图中的绘画感}

摄影术发明之前, 绘画长期担任着复制摹写功能, 本质上是带有叙述性质的对现实的 “客观” 描绘。摄 影与绘画同样作为美的表达，正如宗白华所说: “美 的形式之积极作用是组织、集合、配置。一言蔽之, 是构图。使片景孤境自织成一内在自足的境界, 无待 于外而自成一意义丰满的小宇宙, 启示着宇宙人生的 更深一层的真实。” ${ }^{[2]}$ 两者在构图美上有相似性, 然 而摄影的出现, 挤占了绘画原本职能, 作为新出现的 艺术手段, 不可避免的对长期积淀的绘画进行学习, 尤其是以人物为基础的纪实摄影将绘画的手法融入 到自己的创作语言中, 从而使影像更具有吸引力。《捆 麦子的女人》是吕楠 1999 年在西藏拍摄的捆麦子的 女人, 第一眼看去, 观者便能联想到绘画中米勒的《拾 穗者》油画作品。在画面构图中融入了西方油画的创 作手法, 将人物置于大空间中, 通过人物群组化把两 个人物联系起来, 使画面中人物看上去紧凑而不松散。 画面中人物主体相对较小, 留下大面积的背景刻画, 景象的远、近、中景的处理, 强调了人物与景象的相 互关系, 又使得各自具有独立性。吕楠在摄影画面的 构图中直截了当的寓意了绘画故事性, 画面虽没有绘 画笔触的模仿的处理，依然给观者一种强烈的绘画感 受, 这得益于景别的处理及人物动态刻画进行的视觉 建构, 吕楠正是利用这种视觉表现能力让观者可以从 一群淳朴的农民形象中感悟到如画般的意境。

\section{2 吕楠摄影影调美学}

影调是利用光影变化构成画面具有视觉的节奏 和韵律，不同的影调能够给予观看者不同的审美体验。 吕楠的《四季》通过光线的处理使画面避免平铺直叙 的中间调, 利用高调与低调的冲突而赋予影像以民族 诗意美学。使得观众驻足于照片前时, 可以体验到西 藏农民游走于 “理想” 与现实亦实亦虚的精神追求。 


\subsection{1 质朴的黑色调}

在吕楠的镜头下, 既没有西藏雄伟壮阔的自然风 光, 也没有宗教神圣喇嘛和宏伟的庙宇, 有的只是真 真正正的农民生活。吕楠形容自己的工作并不是在拍 “西藏的农民”，而是在拍真正的“人的生活” — - - 正如每一个观者曾经也经历过的, 但在大多数地 方已经消失的人类健康的质朴的生活。摄影师怀着谦 卑之心拍下每一张照片。尽可能去掉观者、摄影师以 及被摄者之间的歧义，让本质的东西以最根本的方式， 让人更好理解的方式, 或者吸引人的方式, 打动人的 方式, 传达出来。 ${ }^{[4]}$ 吕楠在《挖土豆的老人》中赋予 挖土豆的老人以黑色影调, 光线的刻画使观者的视点 停留在男人额头, 人物形象抽象为坡纹线条, 而这种 抽象线条又因黑色影调得到强化, 转化为了时间磨砺 的具体形象, 图中男人成为时间历程上的标志物。吕 楠正是从这种黑色调出发, 使观者的确能够发现黑白 摄影中的象征性, 画面也显得更为庄重严肃, 一定程 度上给观看者留有更为丰富的想象空间。黑色影调下 的西藏农民形象刻画, 成为农民群像的代表, 是中华 大地上农民质朴性格的具象表现, 他们是现实中生活 的 “黑色” , 压制着自身个性, 用双肩扛起生活的信 念。画面中虽然裉去了农民劳作的辛苦氛围, 依旧可 以从细节展现了现实的艰辛, 吕楠在影像中正是利用 这种黑色调让观者感受到一种现实的压迫感。

\subsection{2 恬静的白色调}

在影集中黑色调是对个体质朴性格的忠实记录 的话, 白色调则是对一种恬静的生活本质的宣扬。不 同于彩色摄影的是, 黑白摄影中影调的变化能够非常 直截了当的影响观者对画面内容的体验, 影调具有将 具象事物高度抽象能力, 当这种抽象运用在纪实摄影 中时, 很自然地成为了一种以抽象写具体的创作手段, 营造出一种与我们眼睛正常视角所能看到的五颜六 色的事物而相对不同的世界, 从而对审美体验形成了

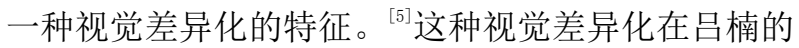
纪实影像中利用白色影调进一步强化, 白色影调本身 具有使观者感受到圣洁、明朗等之意, 加之吕楠影集 拍摄于西藏, 那种宽阔的天地之气, 更赋予了作品一 种难以言说的恬静气息。纪实影像中的农民生活仿佛 已经超脱了物质追求, 成为一种精神的凝练。农民致 力于将自己的信仰播种在这广阔的土地间, 通过他们 的辛勤耕耘得到四季轮回的成果。在《犁地的夫妇》 这幅作品中, 吕楠利用逆光的拍摄手法将人物置于广 阔的天地之间, 农民是沟通天地的 “使者” , 人物自 身的暗调是对现实的塑造, 而大面积白色调的天空则 是他们的 “理想”之地。吕楠在他的影像中用高调的 画面风格将农民的精神世界进行视觉化阐释, 让原本 无法捕捉的细淢情绪跃然于视觉载体之上, 是他透过 简单影调形成与现实世界不同的视觉差异。在白色影 调的祄托下, 整幅作品中生活的本质不是都市中日复 一日的忙碌, 也不是农村无所事事的悠闲, 而是通过 四季劳作之后得到应有成果之后的精神享受。

\section{3 吕楠摄影视觉哲学}

\section{3. 1 多视角的摄影 “意趣”}

罗兰巴尔特总结整理关于图片阐释的摄影理论, 对我们分析图像具有借鉴意义。其中关于摄影 “意趣” 的解释, 在此引用罗兰巴特原文: “意趣属于喜欢而 不是爱的范畴, 它调动起来的是个半吊子欲望……意 趣是一种教养 (知识和礼仪) 类的东西, 能使我认出 操作者, 能使我体会出他那些想法, 那些想法是他进 行拍照活动的依据。” ${ }^{66](336-37)}$ 利用罗兰巴特的摄影“意 趣” 观点分析吕楠这部纪实摄影作品, 会发现影像中 引导观众的摄影 “意趣” 是多重的, 这种视觉感悟不 仅受制于观众不同的生活经验, 还来自于摄影师的创 作手法。在这部纪实摄影作品中, 吕楠的多视角拍摄 方式能够直观的给予观众不同的视觉体验。

他我视角下对西藏农民的客观记录。摄影师吕楠 的他我视角不同于其他摄影师所拍摄的西藏, 将高原 地域的凛洌寒风、粗狂的图腾崇拜、神性的宗教仪式 等标志性的视觉符号排除在画面之外, 而在他的影像 作品中总是记录普通的西藏农民生活的点滴画面, 让 众多观者看到了西藏民众普通生活中不普通的恬静 与自然。 ${ }^{[7]}$ 他我视角下, 摄影师与被摄者始终通过镜 头沟通, 镜头构成了两者之间天然的距离。通过吕楠 的镜头, 观者得以了解这片神秘大地上人们的真实生 活, 打破了人们对西藏原有的固定印象。对于西藏的 认识, 不再是由宗教、高原等元素组成的权威解读, 也不单是质朴与恬静的农村生活, 而是它们共同构成 了对西藏的客观认识。观众在观看影像时, 画面的时 间性被切割, 照片中的西藏农民保持着永恒的姿势。 观众通过照片媒介以第三人称的视角介入画面中人 物生活, 保持着相对客观的审美评价。在此视角下, 摄影师与观众保持相同地位, 是纪实摄影真实性的完 美写照, 能够使影像保持独立性与客观性。

自我视角下对西藏农村的主动观看。当摄影师进 行专题摄影时, 不可避免的主观介入被摄对象的生活, 通过近距离的接触, 使得被记录下来的影像更具有真 实性。而这种主观视角下的纪实影像也对画面空间进 行压缩, 客观记录的属性被主动观看取代, 视角的重 心集中于主动观看的精神解读。吕楠长期潜伏于西藏 农村拍摄, 他与被摄者之间的距离通过长期的融合而 缩短, 在他的镜头下, 我们看不到镜头突击的侵扰, 取而代之的是平静祥和的叙述。摄影师作为西藏农村 农事活动中的一份子, 相机的拍摄框架趋于眼前的主 观视角, 从而使得画面的重心强调农事氛围下的精神 活动, 这种精神活动在画面中以远近透视的动态观看 得以视觉表现。在此视角下, 观众被摄影师的镜头拉 进画面之中，观众如身临其境般同影像中的被摄者进 行精神交流, 从而对画面的感悟不再是单纯的视觉真 实, 而是转化为主动观看视角的下的精神真实。 


\section{3. 2 生命存在的摄影 “刺点”}

罗兰 -巴尔特将 “刺点” 解释为: 它的某处击中 了我, 刺痛了我。即《明室》中阐述的 “我能够说出 名字的东西不可能真正刺激得了我。我不能说出名字, 是思想混乱的明显征兆。……有的时候, 我觉得想到 的照片比看到的照片还清楚, 好像直接看到的东西会 产生误导, 让人努力用语言去描述, 反而总是错过了 效果的那个点, 即刺点。” ${ }^{[6](P 70)}$ 这种刺痛并不是画面 直接的视觉表现的, 实际上是照片中所蕴含的复杂的 对象信息和拍照者个性化的观看态度造成的。吕楠在 这部作品中给予观众的精神体验是多样的, 其中关于 生命存在的 “刺点” 值得我们进一步深挖。

现实中的生命存在。每个生命都有终结之时, 每 个人都畏惧死亡的到来, 但又没有人能阻止这一进程。 吕楠在拍摄《四季》时, 大量使用了如《外祖母和外 孙女》一样的画面布置手法, 将一老一少置于画面之 中。这本身源于东方仪式感的纪念照片形式, 却又不 经意间流露出关于生命存在的思考。照片中的 “刺点” 通过被摄者的皮肤状态得以展现, 历经沧桑的成年人 或者老人有着一份从容不迫的沉稳, 孩童与之相对的 表现出天真烂漫, 当观者在脑海中将时间之钟回拨, 几十年前的老者也如现在的孩童一般。在吕楠的镜头 中我们除了看到了藏族人民生活的痕迹, 他们的形象 更体现出自然性的, 未被工业文明污染的原始状态。 这种原始状态少了很多被艺术需要的苦难痕迹, 而是 “出世气息” 的归隐形象。 ${ }^{[8]}$ 在摄影美学语言中, 这 种正面的描绘意味着永恒, 吕楠正是通过东方仪式感 的正平面描绘将时间性重新引入, 而平面的视觉表现 意味着西藏农民本身生命的存在或本质, 这是不受时 间影响的。

照片中的生命存在。摄影本身是关于 “存在” 的 艺术, 相机的应用源于对逝去的时光以及一去不返的 人物形象的记录, 当相机快门定格照片时, 影像成为 生命存在的概念体。吕楠利用影像将西藏农民家庭进 行定格, 正如在图一中, 老妇人与孩子组成了老少的 生命组合, 照片的定格将两者此时的生命凝固于视觉 媒介之上。观众的观看随着时间的流逝, 虽然无法得 知被摄者是什么样, 照片中的形象与真实的被摄形象 对应不再具有等价性, 但是照片的长久保存将这一生 命存在定格, 得到了关于存在的记录。影像中“刺点” 是个体生命被定格于某一时刻, 观众对被摄者生命的 视觉感受得到延长, 而被摄者本身的生命却依旧走向 生命的终结。以摄影哲学思考生命存在, 这一“刺点” 将观者从生命存在的精神体验拉回到现实世界, 认清 生命的本质, 能够直面生活的困境与磨难, 而不纠结 于生命的短暂与易逝。

\section{3. 结语}

吕楠拿着相机追寻着远离现代文明的精神产物, 在这部作品中以布道者的精神记录着这群被概念模 式化生活方式所忽略或排斥的人群, 利用照片将西藏
农民从生活边缘拉到我们眼前。吕楠说他自己不信仰 宗教, 虽然很多人在他这部作品中看到了神性, 但展 现的更多的是真诚的人性。镜头下的西藏农民无比质 朴, 而又有脱离现实的状态, 吕楠以一种调和的方式 将真诚的精神与恬静的现实结合，把握着两者之间的 共性与平衡, 用影像探讨着西藏农民的生存状态, 关 怀着他们的生存现状, 肩负信仰, 又忠于现实。吕楠 的《四季》作为《被遗忘的人》和《在路上》的精神 “升华”, 是对灵魂的慰藉和对生活本真的向往。他 以影像抒情, 捕捉那些安静的画面, 却不仅仅只是停 留于视觉效果, 最终呈现了摄影师的真诚与西藏农民 的虔诚对话。

\section{REFERENCES}

[1] Yu YJ, Jing M. (2019) Walking between ideal and reality as a "mind seeker" -- A study on the subject characteristics of $\mathrm{Lu}$ Nan's documentary photography Works. J. Journalism Lover., 02:62-64.

[2] Huang YR. (2010) On Photography. Shanghai Translation Publishing House, Shanghai.

[3] Zong BH. (2002) Aesthetics for a walk.Shanghai Renmin University Press, Shanghai.

[4] Wang Y. (2009) Lu Nan trilogy: The return of the great human spirit. J. Learning expo, 04:22-23.

[5] Xie JL. (2013) Interpretation of Aesthetic connotation of Chinese documentary photography. Shanghai Normal University, Shanghai.

[6] Zhao KF. (2011) Camera Lucida:Reflections on photography. China Renmin University Press, Beijing.

[7] $\mathrm{Mu}$ XR. (2016) Analysis and research on documentary photography works of Lu Nan's spiritual essence. J. The mass of literature and art, $11: 166+241$.

[8] Xiong MF. (2019) Research on Lu Nan's documentary photography from the perspective of social memory. Yangzhou University, Yangzhou. 